\title{
Generalized Digital Reassembly using Geometric Registration
}

\author{
Anthousis Andreadis, Georgios Papaioannou and Pavlos Mavridis \\ Department of Informatics, Athens University of Economics and Business \\ Email: \{anthousis, gepap, pmavridis\}@aueb.gr
}

\begin{abstract}
We present a novel and generic user-guided approach for the digital reconstruction of cultural heritage finds from fragments, which operates directly on generic 3D objects. Central to our approach is a three-tier geometric registration approach that addresses the reassembly problem using i) the contact surface of the fractured objects, ii) feature curves on the intact surfaces and iii) partial object symmetries. In contrast to most existing methodologies, our approach is more generic and addresses even the most difficult cases, where contact surface is unusable, small or absent. We evaluate our method using digitized fragments from the Nidaros Cathedral.
\end{abstract}

Keywords-Reassembly, registration, alignment, features, symmetry detection, geometric priors

\section{INTRODUCTION}

The reconstruction of cultural heritage finds is a timeconsuming and difficult task, especially for large objects or large collections of fragments. The digital counterpart, virtual object reassembly, has received significant research interest in the past years, mainly with regard to specialized object types, such as frescos and pottery. This virtual, computer-assisted domain provides numerous advantages, including the access to remotely located physical finds and the ability to easily manipulate 3D shapes, whose physical counterparts may be hard to handle. Above all, it benefits from the exploitation of robust and fast (semi-) automatic algorithms for computing and exhaustively testing hypotheses at a large problem scale, even extreme ones. Interestingly, algorithms and methods developed in the scope of computational archaeology can be also applied to the domains of forensics and computer-assisted surgery, which have also expressed interest in such methods and perform related research.

In computational archaeology, the problem is described as the automatic process that involves the identification of potentially fractured parts/regions of an object, the search for corresponding pieces within a fragment collection and finally the clustering and pose estimation of multiple parts that result in a virtual representation of (partially) reassembled objects. In the general case, the problem has $2 \ldots N$ input part representations (surfaces, volumes, point-clouds etc.), each expressed in its own local coordinate system. The resulting solution consists of $1 \ldots M$ clusters. If no cyclic associations are allowed, this translates to at most $N-M$ rigid transformations that describe the pose of the fragments in the output clusters.

Typically, the process starts with the digitization (e.g. 3D scanning) of the physical fragments and continues with the pre-processing of the fragment geometry in order to extract the fractured and intact surfaces (segmentation and classification).
Subsequently, all pairwise combinations of the fragments are tested for alignment and a matching error is computed. This step usually begins with a global registration process that examines the solution search space for a good but rough alignment, that in turn initiates a local registration process in order to refine the solution. The complete set of the pairwise results drives subsequently the multi-part alignment (reassembly), where complete objects are formed by finding the global position for each fragment.

In this work, we focus mainly on the pairwise and multipart registration steps of the reassembly by introducing a three-tier geometric registration approach that was designed to help expert users during the reassembly process. The main contribution of our approach is the ability to provide plausible solutions for objects with high erosion or even large missing parts, which is usually the case in most cultural heritage scenarios and which almost all existing approaches fail to address.

In the rest of the paper we initially present an overview of existing work in the field, we proceed to elaborate on our three-tier geometric registration approach and finally, we present results of our approach, when applied to actual cultural heritage data from the Nidaros Cathedral in Trondheim.

\section{PREVIOUS WORK}

Depending on the type of the fragments, the general reassembly problem can be specialized according to the following categories:

Two-Dimensional (2D) Reassembly. While all real objects have a third dimension, for certain relatively flat objects, such as frescos and stone tablets, it is safe to make a simplification and reduce the problem to two dimensions, without this affecting the quality of the final reassembly. The work of Leitao and Stolfi [1], Kong and Kimia [2] and Papaodysseus et al. [3] are some of the most representative examples in this category that utilize some form of elastic curve matching to address the problem. Different approaches have also been presented, such as the work of Sağiroğlu and Erçil [4], where texture synthesis and in-painting techniques are used in order to find the solution.

Restricted Three-Dimensional (2.5D) Reassembly. Methods in this category solve the reassembly problem using $3 \mathrm{D}$ objects for which either the actual dimensionality is less than 3 (e.g. contours or surfaces embedded in 3D space) or the degrees of freedom for the matching reduces the pose estimation transformation to a two-dimensional problem. In the literature, the first set of problems usually regards "thin-walled fragments", 
typically pottery sherds. The problem is usually addressed as a specialized case of geometric priors (rotational symmetry), using estimates of the axis and break curves as extracted from the scanned sherds. Typical examples of such methods include the work of Cooper [5], Kampel and Sablatnig [6], Son et al. [7]. The latter problem case targets "flat pieces with thickness", which are thick fresco fragments in their majority. Brown et al. [8] address the problem by uniformly sampling the perimeter (ribbon) of each fragment, constraining the transformations on the 2D plane and using a 3D contour matching approach. Similarly, Belenguer et al. [9] use a shape descriptor on the discretized fracture ribbon and a hierarchical approach.

Three-Dimensional (3D) Reassembly. This is the most general and difficult case of the problem that targets 3D freeform fragments. Several works, in order to avoid the difficult global registration step, employ a semi-automatic approach, where the user drives the reassembly by either specifying constrains or enforcing particular matches. In the work of Parikh et al. [10] the user selects possible pairs from a set of compatible parts and a compatibility score is computed based on local features on the fractured surfaces. In another approach, Mellado et al. [11] propose an interactive loop, where the user provides approximate initial positions for pairwise matches and refine the alignment, using a variant of the well known ICP algorithm [12]. In a more recent approach, Palmas et al. [13], focus on eroded fragments and fractured surfaces without features by employing a distance minimization scheme on a rough alignment given by manually registered landmarks on the two fragments.

Other approaches try to minimize the user intervention by focusing on more automated pipelines. Papaioannou et al. [14] were the first to address the problem, under the assumption of nearly planar surfaces with a good matching area. The matching of the fragments is based on the distance of the fractured facets and the process is accelerated using 3D hardwarebased distance-to-plane queries. Huang et al. [15] proposed another 3D object reassembly method that matches fragments based on multi-scale features of the fractured surfaces. The evaluation of the work is performed with non-cultural heritage objects with rich intrinsic geometric features and no significant erosion. Li et al. [16] and Altantsetseg et al. [17] focus only on the pairwise alignment of fragments, using approaches similar to [15]. While these type of methods perform well on surfaces with rich intrinsic geometric features, cultural heritage fragments may not retain this information due to erosion.

Thuswaldner et al. [18] combine the method of [15] with exploitation of planar surfaces and straight lines in order to achieve alignment of rectangular shaped fragments in the archaeological site of Ephesos. Winkelbach et al. [19] propose an approach that does not rely on features and instead tries to maximize the contact area using a branch-and-bound search heuristic. In a more advanced approach Mavridis et al. [20] propose a 3 -step minimization scheme that uses the $\ell_{p}$ metric with low values of $p$ in order to partially address noise and outliers.

The current work combines a contact surface-based approach [20] with salient feature curves from the intact surfaces of the fragments and further extents the pipeline by exploiting partial symmetries in order to address cases with significant erosion or even missing and disjoint parts.

\section{METHOD OVERVIEW}

As with most reassembly approaches, our data require an initial processing in order to extract the potentially fractured and intact facets of the fragments. Subsequently, the first tier of geometric registration is applied in order to form pairwise matches with good contact area. The resulting pairs can be evaluated by the expert user who is responsible to either accept or discard them. At this point, the user can initiate the second tier of geometric registration for fragment pairs with similar characteristics, but with significant erosion or missing information in their matching fractured facets. Using an intuitive and trivial approach, the user can quickly extract salient feature curves on the intact surfaces of the fragments. These feature curves are next used in an automatic alignment process of the pairs. Having generated the set of matching fragment pairs, the multi-part reassembly procedure is then initiated. Here, the set of matching pairs is explored and objects consisting of multiple fragments are generated.

It is possible that during this process some fragments may remain isolated, either because they form bonds with high error due to incompatible contact surfaces or external features or because they are not directly coupled with the rest of the fragments (disjoint). In order to address this issue, when appropriate, we utilize partial object symmetries in order to extract the complementary geometry of a cluster already formed by

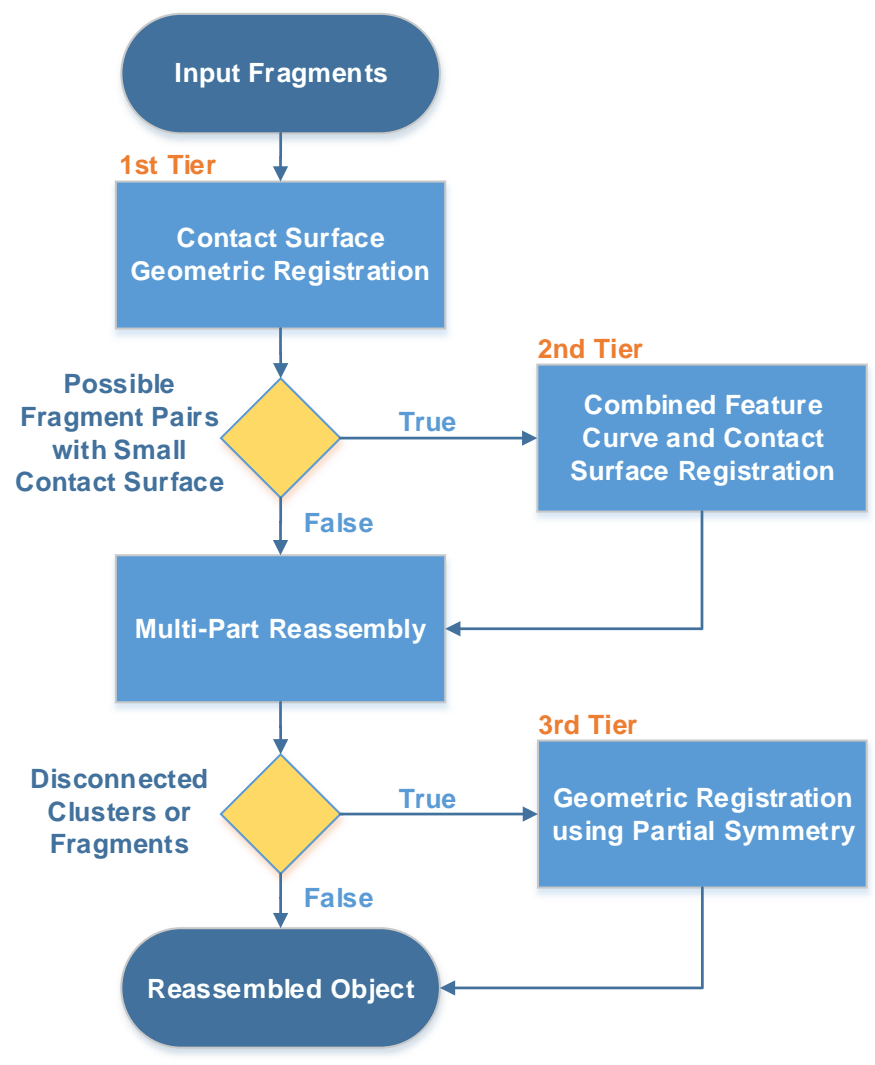

Fig. 1. Flow chart of the proposed three-tier geometric reassembly pipeline. Decision points reflect intervention by an expert user. 


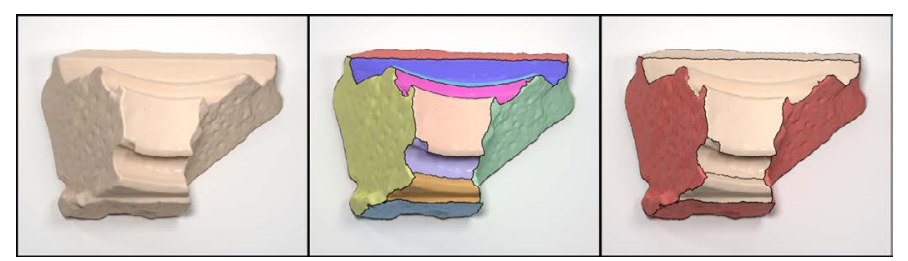

Fig. 2. From left to right, input fragment, segmentation result with colorized segments for clarity and the resulting classification of segments, where red denotes the fractured ones.

the first two tiers. While the symmetrically expanded shape by itself is of little scientific value to the archaeologists, in the third tier of our geometric registration approach, we use it as a guide in order to generate possible registration poses of the disjointed fragments, which can be afterwards evaluated by the experts.

The outline of the above three-tier reassembly pipeline is presented in Figure 1. In the rest of the section we present the processing of our data and each of the proposed stages of geometric registration.

\section{A. Fragment Processing}

As mentioned earlier, in this step the main goal is to extract distinct contiguous surface regions (facets) from the scanned fragments and classify them as fractured or intact. This pre-processing of the input data is performed primarily for robustness, since pairwise matching operations between flat intact surfaces will always yield trivial, yet undesirable matching results. It also increases efficiency, as the exclusion of the intact facets from the geometric comparisons reduces both the search space and the distance measurement operations performed.

We initially extract distinct facets of the fragmented objects using a straightforward region-growing segmentation algorithm with the deviation of surface normals in local neighborhoods as the stopping criterion. The resulting segmentation is reevaluated in a cleanup step, in order to eliminate small segments. Subsequently segments are classified as intact and fragmented using a similar approach to [15] (see Figure 2). While more sophisticated segmentation approaches exist, the robustness of the registration methodology presented in the following sections, does not require a perfect segmentation. Furthermore typical 3D scans of fractured objects are of very high resolution and the segmentation process should be efficient in order for it to be practical.

\section{B. First Tier - Contact Surface Registration}

The first tier of our geometric registration approach is an automatic process, whose goal is to find all matching fragment pairs sharing a significant contact surface, given the complete list of fractured facets per fragment. While this problem has received substantial scientific interest, human evaluation of the results is still crucial, since frequently, nonmatching fragments can be successfully registered, given that the registration algorithm has to be robust to noise and outliers in order to handle surface erosion and scanning defects.

We address this step and the underlying optimization problem using the coarse-to-fine search strategy of Mavridis

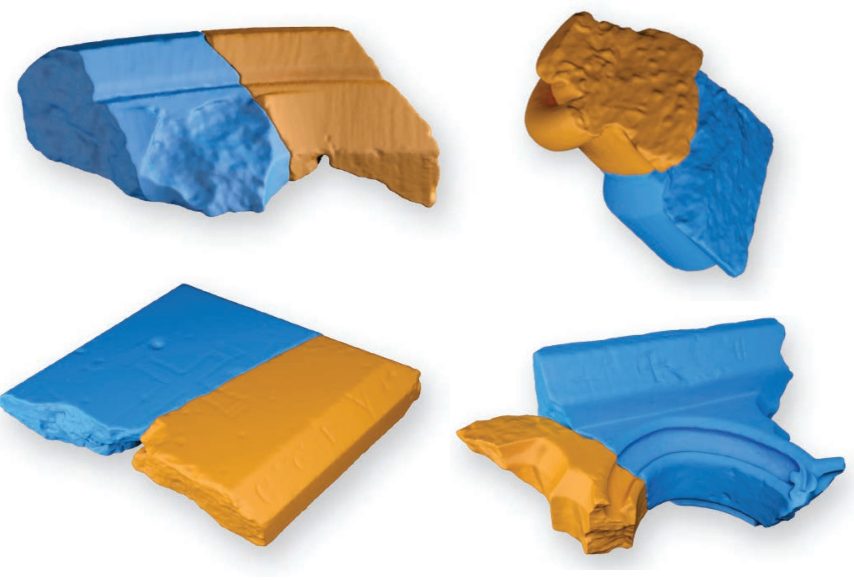

Fig. 3. Pairwise matches obtained using the first tier: contact surface registration. Fragments are colorized for visual clarity.

et al. [20], [21] that is both efficient and robust. The method starts by performing a coarse initial alignment using either a RANSAC-based alignment procedure or the centroids and average normals of the facets depending on the ratio of the surface area of the two facets under investigation. Subsequently, the alignment transformation is parameterized and the search space of the complete set of variables is examined using a Simulated Annealing process, that avoids local minima. Finally, the registration between the two facets is locally refined using the Sparse ICP [22] method and the matching error is reported.

The results are initially trimmed based on the matching error reported and the remaining solution set is either accepted or rejected by the expert user before proceeding to the next step.

\section{Second Tier - Feature Curve Object Registration}

The second tier of the proposed geometric registration approach is a semi-automatic user-guided process that targets the pairwise registration between fragments with significant erosion or large missing parts, i.e. pairs sharing minimal contact surface, where contact surface-based registration approaches fail (see Figure 4).

Due to the nature of this problem, we require the input of an expert, who initially identifies fragments with similar characteristics that could be potentially matching and subsequently,
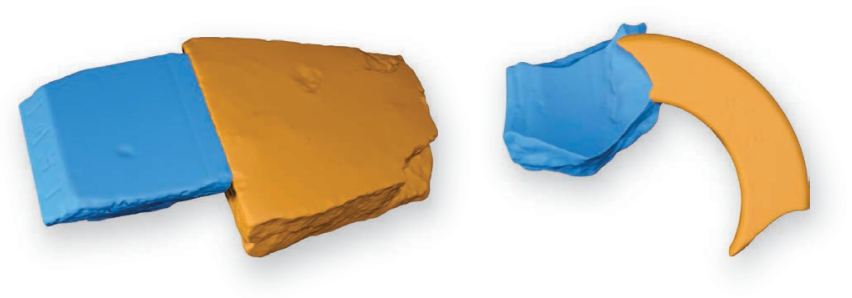

Fig. 4. Failure cases of the surface-based registration. One is rejected as it causes penetration during the multi-part registration stage (left) while the other is rejected by the expert user (right). 


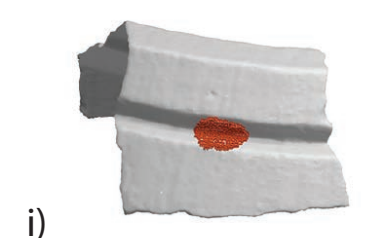

ii)
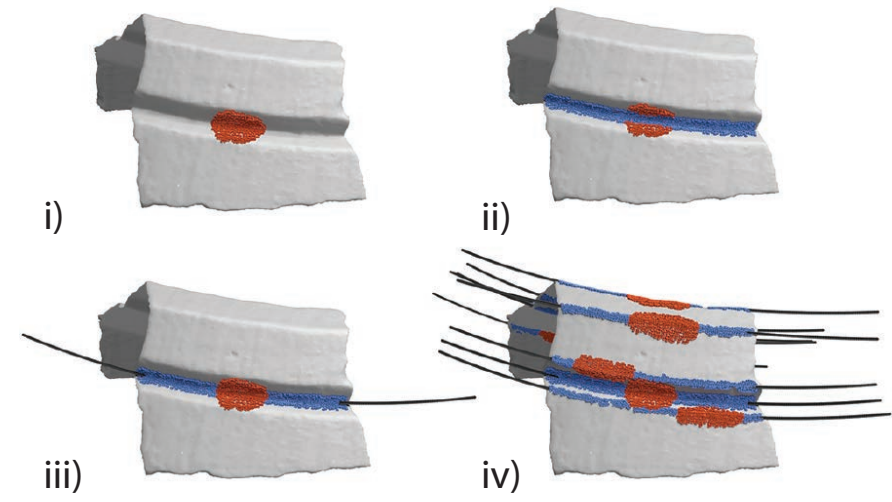

Fig. 5. Salient feature curve extraction process. i) User selects with a brush tool the set of points containing the desired feature curve, ii) Feature points are automatically extracted, iii) Feature curve with extrapolated data, d) Complete set of selected points and extracted feature curves.

using an intuitive and interactive process, extracts the salient feature curves of the fragments.

The feature extraction process is performed in a visual manner, where the user selects with a brush tool a small portion of the fragment that includes part of the desired salient feature. Using mean curvature at multiple scales as a local descriptor, we find the local minima and maxima of the descriptor and extract the points of interest that belong to the salient feature. Subsequently, we perform a thinning process using the skeletonization method of Huang et al. [23] and the resulting point set is approximated using a parametric curve (B-spline) via least-squares fitting. Using curve extrapolation, neighbour points belonging to the same feature are iteratively extracted, until no more points can join the feature set. The user has to repeat the process for each disconnected salient feature curve of the fragment (see Figure 5) and the resulting set of feature curves is used in the following registration process.

We extend the surface-based alignment approach by Mavridis et al. [20] to include in the same minimization scheme the alignment score of the detected salient feature curves. In particular, the distance between feature curves associated with the facet under examination on one fragment and the extrapolated feature curves on the other fragment and vice versa is measured and simultaneously optimized along with the corresponding contact surface (see Figure 6). The two distinct terms, the surface metric $\mathcal{F}_{\text {surf }}$ and the feature curve metric $\mathcal{F}_{\text {curve }}$, are combined in a complementary form:

$$
\underset{\mathbf{M}}{\arg \min }\left(c \cdot \mathcal{F}_{\text {surf }}+(1-c) \cdot \mathcal{F}_{\text {curve }}\right),
$$

where $c$ is the relative contribution of the fracture surface versus the feature curves and $\mathbf{M}$ is the rigid transformation matrix that aligns the fragments. Weight parameter $c$ should be set roughly proportional to the expected contact area of the two surfaces.

The main advantage of this unified approach is that, by exploiting the extracted feature curves, highly eroded or chipped fragments can be registered and aligned robustly. As in the first

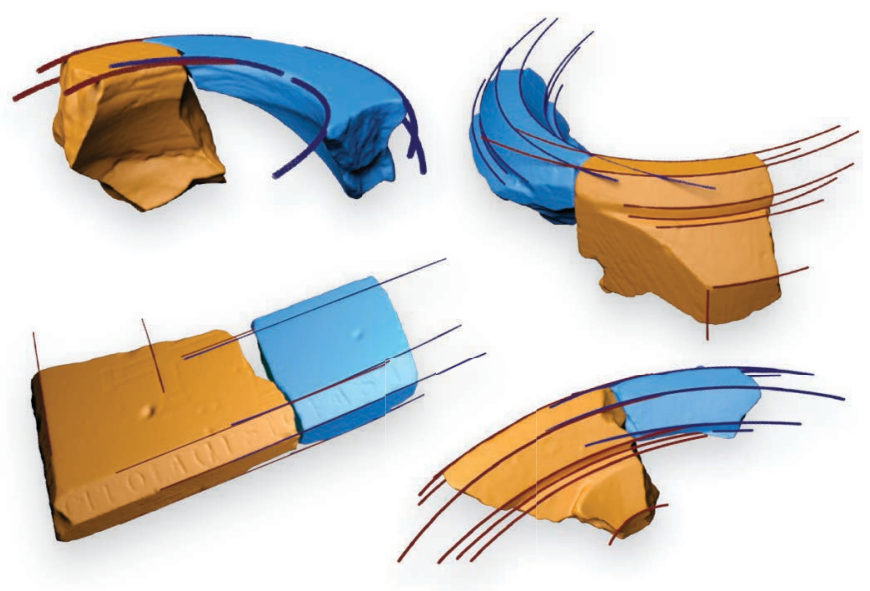

Fig. 6. Pairwise matches obtained using the second tier, with the combined feature curve and contact surface registration.

tier, results are trimmed based on the matching error and the plausible solutions are presented to the expert for evaluation.

\section{Multi-Part Reassembly}

Given the matches and respective matching errors generated in the pairwise alignment stages, in this step of the reassembly, we compute the set of fragment clusters and corresponding global transformations of the fragment meshes. In essence, the mult-part reassembly is performed using a graphbased approach, inspired by the work of Huber [24], where fragments are represented as nodes and pairwise matches as edges with the matching error as edge weight. The optimal set of connections is located by extraction of the Minimum Spanning Forest using the well-known Kruskal's algorithm, with the addition of penetration tests and a back-tracking scheme in order to avoid erroneous results. Finally, the method applies an iterative multi-part local registration step in order to diffuse the propagated error due to slight shifts in the chain of applied pairwise rigid transformations.

\section{E. Third Tier - Symmetry-based Fragment Registration}

The multi-part reassembly step produces objects that are the combination of multiple pairwise alignments, but often, some fragments or clusters of fragments are completely disconnected from the reassembly due to missing parts, bad contact area or the absence of feature curves. In order to address those cases, we propose a novel approach that exploits object symmetries in order to guide the placement of the remaining fragments.

While symmetry detection is a well-researched area, only a few of the methods focus on objects with large missing data. Sipiran et al. [25] use a surface function based on heat diffusion and a partiality-aware voting algorithm to address the problem, even in the challenging case with large missing parts. The method essentially locates the best candidate planes of symmetry on the object and using them one can fill the missing geometry as shown in the application examples of [25].

Since computer-generated, "predicted" information of the missing geometry is of little interest to the cultural heritage community, we steer the results of symmetry-based completion 


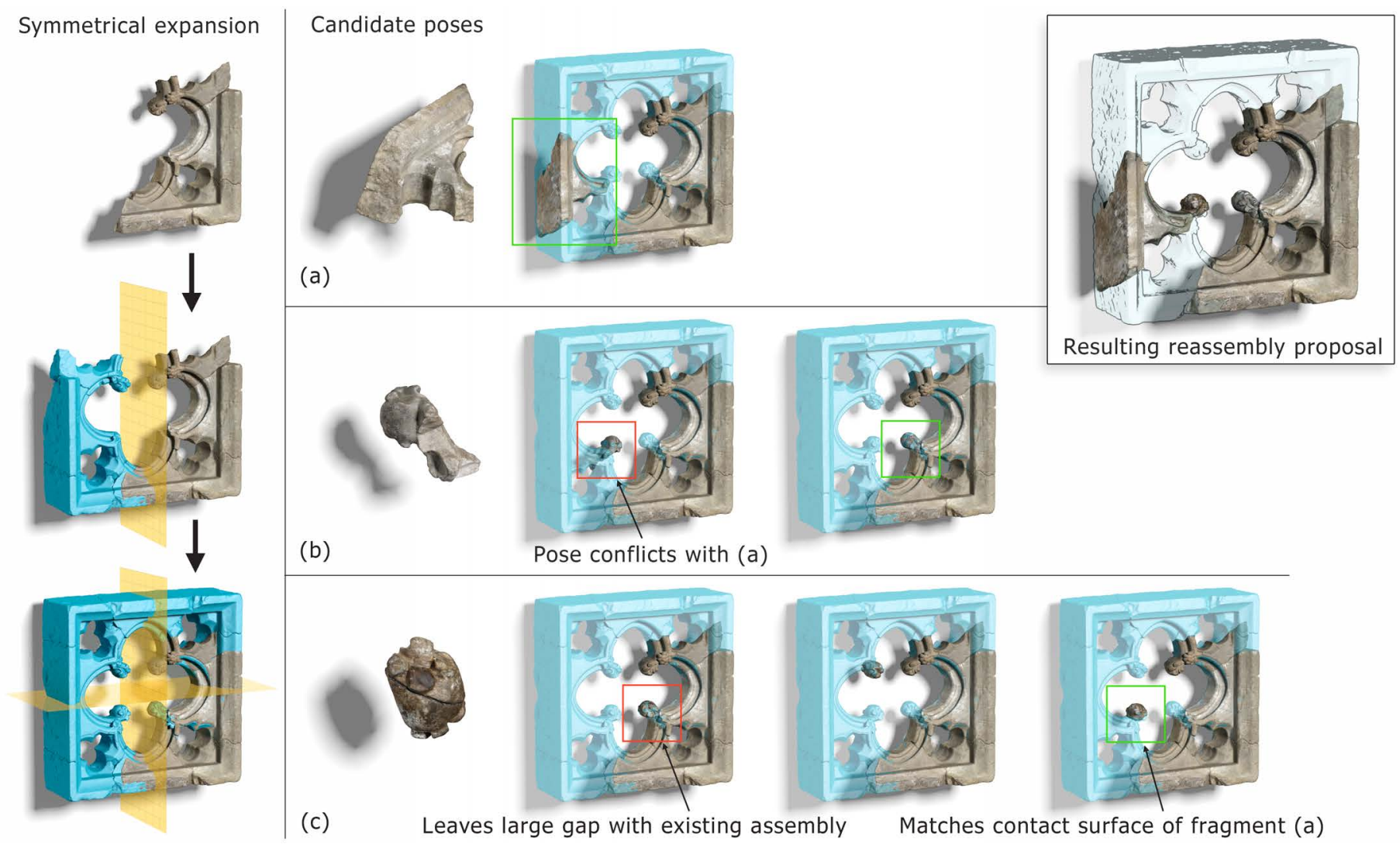

Fig. 7. Third tier registration: Symmetry-based fragment registration. Left: using the partial reassembly, we extract a symmetrical expansion of the object. Middle and right: Potential candidate poses for the disjoint parts (a),(b) and (c) are generated and filtered by the CH expert, to result in the final reassembly of the object.

to a usable direction; using either the method of [25], or even user-supplied planes of symmetry, under a symmetrical interpretation of the evolving shape, we generate only the missing geometry of an object and use it as a geometric prior (guide shell) to match and align any disconnected fragments. Thus, the symmetrical expansion of the assembly generated so far is never included in the final solution but rather only assists our system in order to suggest potential matches of disjoint fragments, which are inspected by the $\mathrm{CH}$ expert. The whole processes is demonstrated in the example of Figure 7.

In this registration step, we follow a different surfacebased search strategy. The 4PCS method of Aiger et al. [26] was designed to perform robustly in the presence of extensive outliers, using four-coplanar point bases to detect hypotheses (potential alignments) and a RANSAC-style search strategy. Due to the exhaustive coverage of the search space and its linear time complexity, the method is impractical, but its recent improvement (Super4PCS) by Mellado et al. [27] reduces the time complexity to quadratic and presents a good compromise between efficiency and robustness.

Using Super4PCS, we generate all the possible registration solutions and sort them according to their registration score. We filter out similar results and present to the user the $k$-best registrations (where $k$ is specified by the user), after refining them using Sparse ICP. Let us note that, while in the general registration case, Super4PCS requires as input the expected overlap, here we are solving a more specific problem (part-in- whole matching) and this specific parameter, is automatically generated using the ratio of the diagonals of the bounding boxes of the two objects.

\section{RESUlTS}

In order to evaluate our proposed methodology, we performed several experiments with real cultural heritage data, a subset of which is presented throughout our figures.

Pairwise alignment results with fragments that share good contact areas can be seen in Figure 3. These results were obtained with the first-tier contact surface registration, without user input, using the same optimization step parameters for the entire collection. Experiments were performed on a Core i7-3820 processor and on average, each facet combination required 0.6 seconds.

Using the same parameters in Figure 4 we see failure cases of the first tier registration, due to the small contact area between the fragments. Such fragment pairs can be successfully aligned, using the second-tier registration approach that utilizes both contact surface and salient feature curves extracted from the intact surfaces. Successful registrations of fragment pairs with small contact area shown in Figure 6. The two left results were obtained primarily with the feature curves, using low values of $c$ parameter (see Equation 1) as the contact area of the fragments is not reliable. In contrast, in the two right examples the contribution of the two terms was almost equal. 

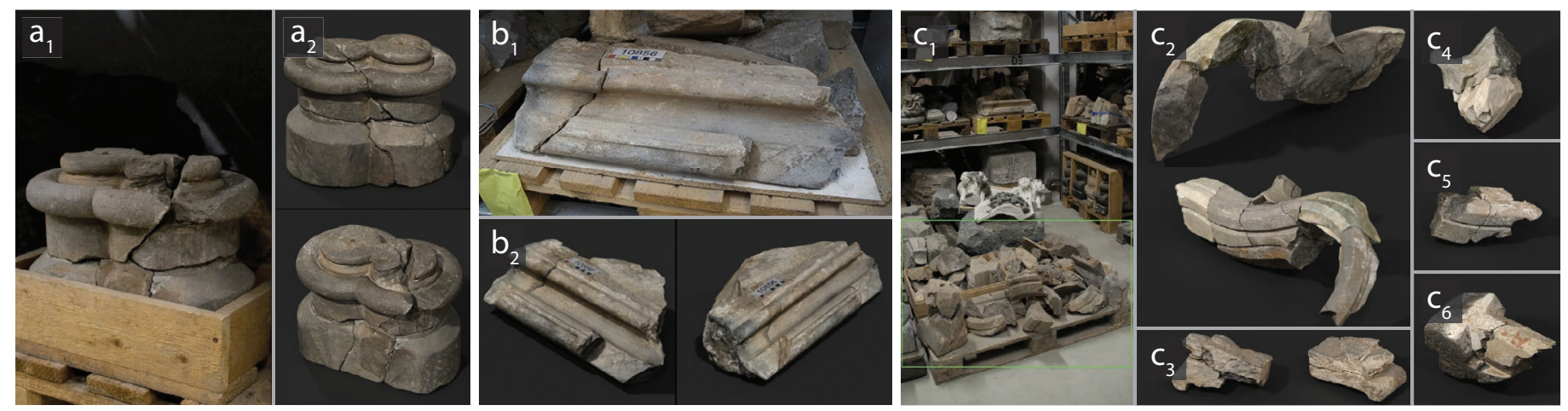

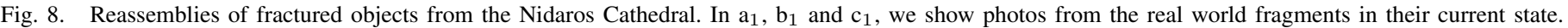

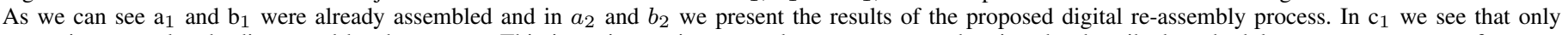

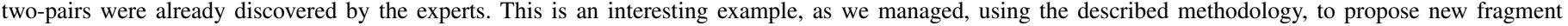
pairs and assemblies as seen in $\mathrm{c}_{2}, \mathrm{c}_{3}, \mathrm{c}_{4}, \mathrm{c}_{5}$ and $\mathrm{c}_{6}$.

While the second-tier approach requires more initial processing for the extraction of the salient feature curves, the optimization step is of similar complexity to the first tier and we observed similar times for the computation of each facet combination. This is due to the fact that curve-to-curve distance is measured using a regular point sampling; curves are first converted to a point representation (point sets) and distances are then evaluated with fast point to point set queries, accelerated via a $k$-d tree data structure.

Complete object reassemblies are presented in Figure 8, where we present photos from the real world fragments alongside the achieved reassembly. The first two results $\left(a_{2}\right),\left(b_{2}\right)$ and a subset of pairwise alignments from the third $\left(\mathrm{c}_{3}\right),\left(\mathrm{c}_{4}\right)$ were obtained using solely pairwise alignments generated with the first tier registration and the multi-part reassembly step we described earlier, without any user input. Most of the results in the third example, $\left(\mathrm{c}_{2}\right),\left(\mathrm{c}_{5}\right)$ and $\left(\mathrm{c}_{6}\right)$, were obtained using alignment with both contact surface and feature curves. As we can see from the photograph of the fragment heap, many pairwise results, as also the large reassembled part obtained with our method, had not been discovered prior to the digital reassembly process, mainly due to the small contact area shared between fragments and the large number of structurally similar pieces.

Finally, in Figure 7 we showcase the use of our thirdtier symmetry-based registration on another fractured object. The multi-part reassembly step resulted in one large cluster of fragments, two disconnected fragments and one small cluster of two fragments that was also disconnected from the rest of the reassembly. On the left of the figure the large cluster is shown, along with evolution of the predicted shape of the entire object. To this end, the expert's hypothesis of a symmetrical shape enabled the exploitation of two planes of symmetry to automatically generate a geometric expansion of the virtual object and use it as a guide to align the disjoint fragments. The symmetrical, complementary expansion can be generated either by the user or by an automated process (in this example, the expert user). This complementary part is subsequently used, in order to find plausible positions for the disconnected fragments as shown in (a) and (b) and (c). It is crucial to note here that this process is mainly guided by the $\mathrm{CH}$ expert as explained in detail in the figure. In this particular example, part (a) was uniquely matched with significant overlap with the complementary shape only in one position. For part (b), two proposals with significant overlap were generated by the modofied Super4PCS approach. The first conflicted with the unique solution for part (a) due to penetration and was rejected. The second placement of part (b) (outlined in green) was also found compatible with the existing geometry of the input reassembly. Finally, for part (c), three positions were suggested: the first leaves a large gap between its fractured surface and the corresponding fracture on the existing reassembly, while the other two do not cause any problems. However, the third solution is also validated for match with part (a) and is accepted as the prevailing pose.

\section{CONCLUSiON}

We presented a novel and generic user-guided approach for the reconstruction of cultural heritage finds. Our threetier geometric registration methodology, automatically solves cases, where fragments share good contact area and provides useful tools for the hard cases, where contact area is unusable, small or absent. The user plays an important role in the process, evaluating results at every stage of the pipeline and guiding the reassembly, when there is no sufficient information for an automated procedure. Note that this manual intervention occurs only at large iteration cycles of the method and does not require constant feedback. The evaluation of the proposed approach with real world cultural heritage artefacts shows the effectiveness of our approach even for scenarios that most existing approaches fail to address. As a future work, we intent to focus our research on more automated approaches for the salient feature curve extraction and the symmetry-based registration, in order to make the process even more timeefficient for the $\mathrm{CH}$ experts.

\section{ACKNOWLEDGMENTS}

We thank Ivan Sipiran for providing the symmetry detection tool of [25] and the authors of SparseICP and Super4PCS algorithms for the publicly available implementation of their research work. Finally we would like to thank Nidaros Cathedral for the data-set [28]. This work was supported by EC FP7 STREP project PRESIOUS, grant no. 600533. 


\section{REFERENCES}

[1] H. da Gama Leitao and J. Stolfi, "A multiscale method for the reassembly of two-dimensional fragmented objects," Pattern Analysis and Machine Intelligence, IEEE Transactions on, vol. 24, no. 9, pp. 1239-1251, Sep 2002.

[2] W. Kong and B. Kimia, "On solving 2D and 3D puzzles using curve matching," in Computer Vision and Pattern Recognition, 2001. CVPR 2001. Proceedings of the 2001 IEEE Computer Society Conference on, vol. 2, 2001, pp. II-583-II-590 vol.2.

[3] C. Papaodysseus, T. Panagopoulos, M. Exarhos, C. Triantafillou, D. Fragoulis, and C. Doumas, "Contour-shape based reconstruction of fragmented, 1600 bc wall paintings," Signal Processing, IEEE Transactions on, vol. 50, no. 6, pp. 1277-1288, Jun 2002.

[4] M. c. Sağiroğlu and A. Erçil, "A texture based approach to reconstruction of archaeological finds," in Proceedings of the 6th International Conference on Virtual Reality, Archaeology and Intelligent Cultural Heritage, ser. VAST'05. Aire-la-Ville, Switzerland, Switzerland: Eurographics Association, 2005, pp. 137-142.

[5] A. Willis and D. Cooper, "Bayesian assembly of 3D axially symmetric shapes from fragments," in Computer Vision and Pattern Recognition, 2004. CVPR 2004. Proceedings of the 2004 IEEE Computer Society Conference on, vol. 1, June 2004, pp. I-82-I-89 Vol.1.

[6] M. Kampel and R. Sablatnig, "Profile-based pottery reconstruction," in Computer Vision and Pattern Recognition Workshop, 2003. CVPRW '03. Conference on, vol. 1, June 2003, pp. 4-4.

[7] K. Son, E. B. Almeida, and D. B. Cooper, "Axially symmetric 3D pots configuration system using axis of symmetry and break curve," in Computer Vision and Pattern Recognition (CVPR), 2013 IEEE Conference on. IEEE, 2013, pp. 257-264.

[8] B. J. Brown, C. Toler-Franklin, D. Nehab, M. Burns, D. Dobkin, A. Vlachopoulos, C. Doumas, S. Rusinkiewicz, and T. Weyrich, "A system for high-volume acquisition and matching of fresco fragments: Reassembling theran wall paintings," ACM Trans. Graph., vol. 27, no. 3 , pp. 84:1-84:9, Aug. 2008.

[9] C. Sanchez Belenguer and E. V. Vidal, "Archaeological fragment characterization and 3D reconstruction based on projective gpu depth maps,' in Virtual Systems and Multimedia (VSMM), 2012 18th International Conference on. IEEE, 2012, pp. 275-282.

[10] D. Parikh, R. Sukthankar, T. Chen, and M. Chen, "Feature-based part retrieval for interactive 3D reassembly," in Proceedings of the Eighth IEEE Workshop on Applications of Computer Vision, ser. WACV '07. Washington, DC, USA: IEEE Computer Society, 2007, pp. 14-.

[11] N. Mellado, P. Reuter, and C. Schlick, "Semi-automatic geometrydriven reassembly of fractured archeological objects," in Proceedings of the 11th International Conference on Virtual Reality, Archaeology and Cultural Heritage, ser. VAST'10. Aire-la-Ville, Switzerland, Switzerland: Eurographics Association, 2010, pp. 33-38.

[12] P. Besl and N. D. McKay, "A method for registration of 3-d shapes," Pattern Analysis and Machine Intelligence, IEEE Transactions on, vol. 14, no. 2, pp. 239-256, Feb 1992.
[13] G. Palmas, N. Pietroni, P. Cignoni, and R. Scopigno, "A computerassisted constraint-based system for assembling fragmented objects," in Proc. of Digital Heritage 2013 International Congress, vol. 1. IEEE, 2013, pp. 529-536.

[14] G. Papaioannou, E.-A. Karabassi, and T. Theoharis, "Virtual archaeologist: Assembling the past," IEEE Computer Graphics and Applications, vol. 21, pp. 53-59, 2001.

[15] Q.-X. Huang, S. Flöry, N. Gelfand, M. Hofer, and H. Pottmann, "Reassembling fractured objects by geometric matching," ACM Trans. Graph., vol. 25, no. 3, pp. 569-578, Jul. 2006.

[16] Q. Li, M. Zhou, and G. Geng, "Pairwise matching of 3D fragments," in Information Management, Innovation Management and Industrial Engineering (ICIII), 2012 International Conference on, vol. 3. IEEE, 2012, pp. 479-482.

[17] E. Altantsetseg, K. Matsuyama, and K. Konno, "Pairwise matching of 3D fragments using fast fourier transform," The Visual Computer, vol. 30, no. 6-8, pp. 929-938, 2014.

[18] B. Thuswaldner, S. Fl"ory, R. Kalasek, M. Hofer, Q.-X. Huang, and H. Th"ur, "Digital anastylosis of the octagon in ephesos," Journal on Computing and Cultural Heritage, vol. 2, pp. 1:1-1:27, July 2009.

[19] S. Winkelbach and F. Wahl, "Pairwise matching of 3D fragments using cluster trees," International Journal of Computer Vision, vol. 78, no. 1, pp. 1-13, 2008.

[20] P. Mavridis, A. Andreadis, and G. Papaioannou, "Fractured object reassembly via robust surface registration," in Eurographics 2015 (Short Papers track). Eurographics Association, May 2015.

[21] _ _ "Efficient Sparse ICP," Computer Aided Geometric Design (Proc. Geometric Modeling and Processing), pp. 16-26, 52015.

[22] S. Bouaziz, A. Tagliasacchi, and M. Pauly, "Sparse iterative closest point," Computer Graphics Forum (Symposium on Geometry Processing), vol. 32, no. 5, pp. 1-11, 2013.

[23] H. Huang, S. Wu, D. Cohen-Or, M. Gong, H. Zhang, G. Li, and B. Chen, "L1-medial skeleton of point cloud," ACM Trans. Graph., vol. 32, no. 4, pp. 65:1-65:8, Jul. 2013.

[24] D. F. Huber, "Automatic three-dimensional modeling from reality," $\mathrm{Ph} . D$. dissertation, Carnegie Mellon University, Pittsburgh, PA, USA, 2002, aAI3076866.

[25] I. Sipiran, R. Gregor, and T. Schreck, "Approximate symmetry detection in partial 3D meshes," Computer Graphics Forum (proc. Pacific Graphics), vol. 33, pp. 131-140, 2014.

[26] D. Aiger, N. J. Mitra, and D. Cohen-Or, "4-points congruent sets for robust surface registration," ACM Transactions on Graphics, vol. 27, no. 3, pp. \#85, 1-10, 2008.

[27] N. Mellado, D. Aiger, and N. J. Mitra, "Super 4pcs fast global pointcloud registration via smart indexing," Computer Graphics Forum, vol. 33, no. 5, pp. 205-215, 2014.

[28] T. Theoharis and G. Papaioannou, "PRESIOUS 3D cultural heritage fragments," 2013. [Online]. Available: http://presious.eu/resources/**3d-data-sets 